\title{
On-Chip Gel-Valve Using Photoprocessable Thermoresponsive Gel
}

\author{
Keitaro Ito $^{{ }^{* *}}$, Shinya Sakuma ${ }^{2 \dagger}$, Yoshiyuki Yokoyama ${ }^{3}$ and Fumihito Arai $^{1}$
}

\begin{abstract}
Microfluidic chips are powerful tools for biochemical experiments. High speed and precise flow control can be achieved by using microvalves on a chip. Several types of microvalves that can be integrated into a microfluidic chip have been reported. Among them, gel microvalves have certain advantages over other valves because of their soft structure, which will contribute to prevent mechanical damage the cells passing though the valve. Here we use Bioresist, a photoprocessable thermoresponsive gel, as a key component of the microvalve. Since Bioresist is photopatternable, we can create any arbitrary 2D shape from the thermoresponsive gel using photolithography. Moreover, Bioresist has the unique feature of a phase transition around $30^{\circ} \mathrm{C}$, and swells and shrinks repeatedly with temperature change. By integrating the patterned thermoresponsive gel with a microheater, we developed a gel actuator and designed a gel-valve. The gel-valve has the advantages of a simple actuation mechanism: high leakage pressure, high speed actuation and low power consumption. The valve is biocompatible and easily integrated into a chip by using conventional photolithography. Using this valve, we achieved on-chip flow control, and applied it to cell sorting on a chip.
\end{abstract}

Keywords: MEMS; Microvalve; Microfluidic chip; Microfluidic control; Thermoresponsive gel; Cell sorter

\section{Background}

Demand for single cell analysis has increased with progress in cell biology and micro- and nanofabrication technologies [1-3]. For the purpose of single cell analysis, single cell manipulation capabilities, such as positioning, immobilization, and sorting of target cells, are needed. Microfluidic chips are powerful tools that make it possible to perform these tasks with high throughput, low cost, and high repeatability [4-8]. Since these cell manipulations are carried out along with flow control, microvalves are key components. For high speed and precise flow control, microvalves should be closely connected to the microfluidic channels on a chip [9-24]. For this purpose, there have been several types of microvalve reported that can be integrated with microfluidic chips, including electrostatic microvalves [9-11], pneumatic microvalves [12-14], magnetic microvalves [15-18], and thermoresponsive gel microvalves [19-25].

Gel microvalves have certain advantages over other types because they have a soft structure, which can prevent

\footnotetext{
* Correspondence: ito.keitaro@biorobotics.mech.nagoya-u.ac.jp

${ }^{\dagger}$ Equal contributors

${ }^{1}$ Nagoya University, Nagoya, Japan

Full list of author information is available at the end of the article
}

mechanical damage to cells passing through the valve. Here we use a photoprocessable thermoresponsive gel (Bioresist, Nissan Chemical Industries Ltd.), as a key component of a gel microvalve. Since Bioresist can be patterned using photolithography, we can create any arbitrary 2D shape from the thermoresponsive gel. Moreover, Bioresist has the unique feature of a phase transition around $30^{\circ} \mathrm{C}$, and swells and shrinks repeatedly with temperature change. By integrating the patterned thermoresponsive gel with a microheater, we developed a gel actuator and designed a gel-valve. The gel-valve is controlled by a microheater, and therefore, its structure is quite simple. The gel-valve has the advantages of a simple actuation mechanism: high leakage pressure, high-speed actuation and low power consumption. The valve is biocompatible and easily integrated into a chip using conventional photolithography. In this study, we focused on the evaluations of the gel-valve using Bioresist as an on-chip microfluidic component. We evaluated the resolution of patterning and expansion rate of Bioresist. After fabrication of microvalves, we evaluated the frequency characteristics and pressure resistance of the microvalves. Finally, we demonstrated on- 

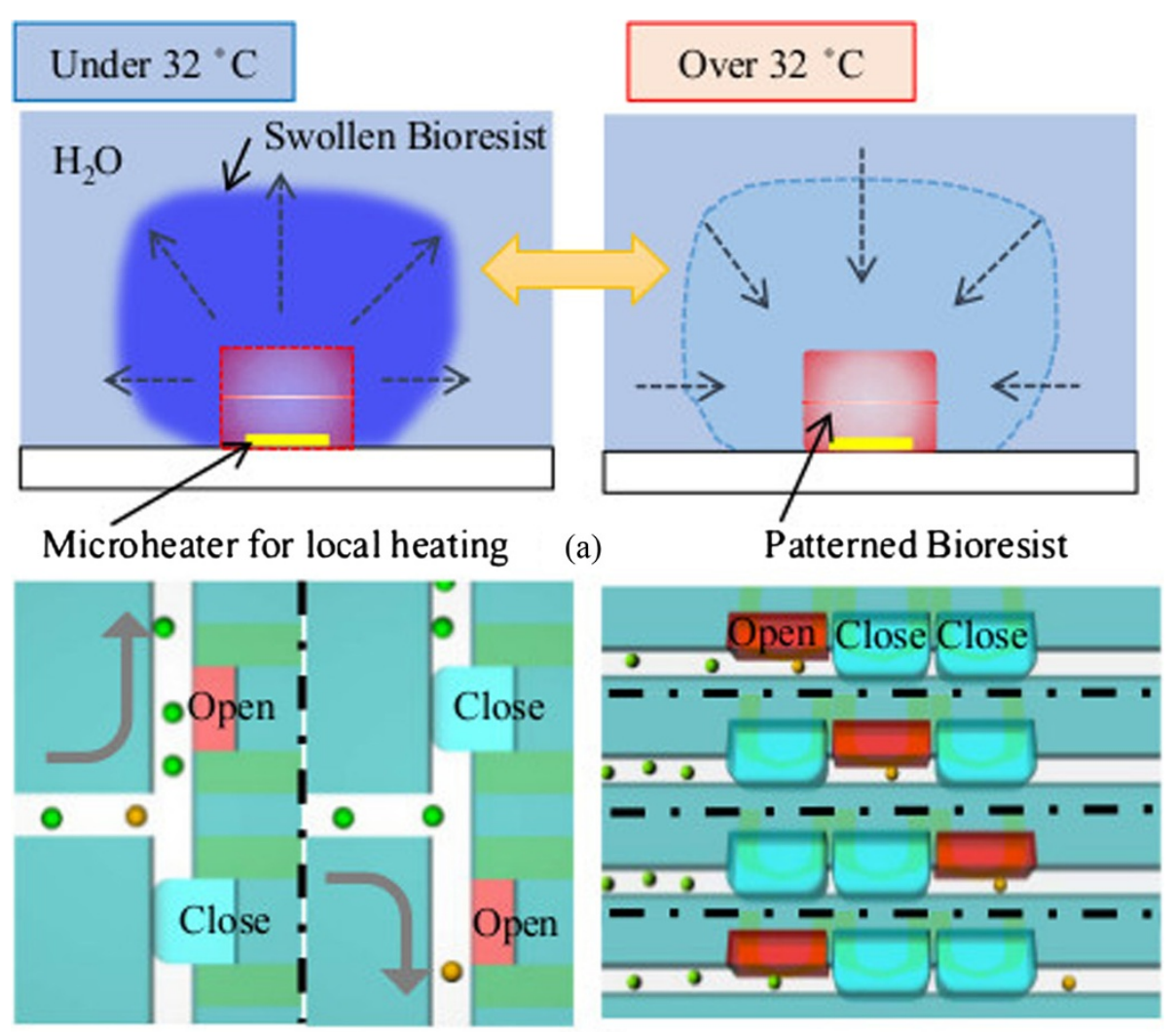

(b)

Figure 1 Basic image of gel-valves. (a) A concept of the on-chip gel-valve. (b) Concepts of sorting and pump using gel-valves.

chip cell sorting as an application of the microvalves by sorting cells using the proposed gel-valves.

The remainder of this paper is organized as follows. After an introduction of microvalves for on-chip flow control, we propose the gel-valve in section 2 . In section 3, we explain the experimental system and results. We discuss advantages of the proposed gel-valve in section 4 , followed by concluding remarks in section 5 .

\section{Methods}

\section{Related works of microfluidic valves}

Many studies of on-chip microvalves have been previously reported. Electrostatic microvalves can be fabricated by silicon-based micro electro mechanical system (MEMS) processes and easily integrated into microfluidic chips [9-11]. For single cell applications, however, they have possibility of mechanical damage to cells passing through (a)

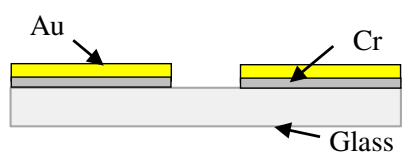

(b) (c)

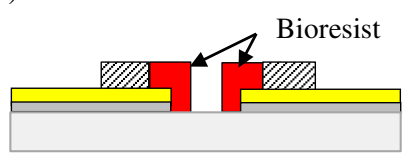

(d)

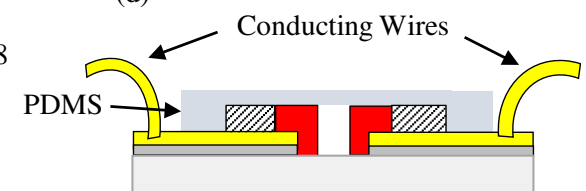

Figure 2 Fabrication process of gel-valve. (a) Patterning of electrode (b) Patterning of SU-8 (c) Patterning of Bioresist (d) Bonding of PDMS and wiring. 


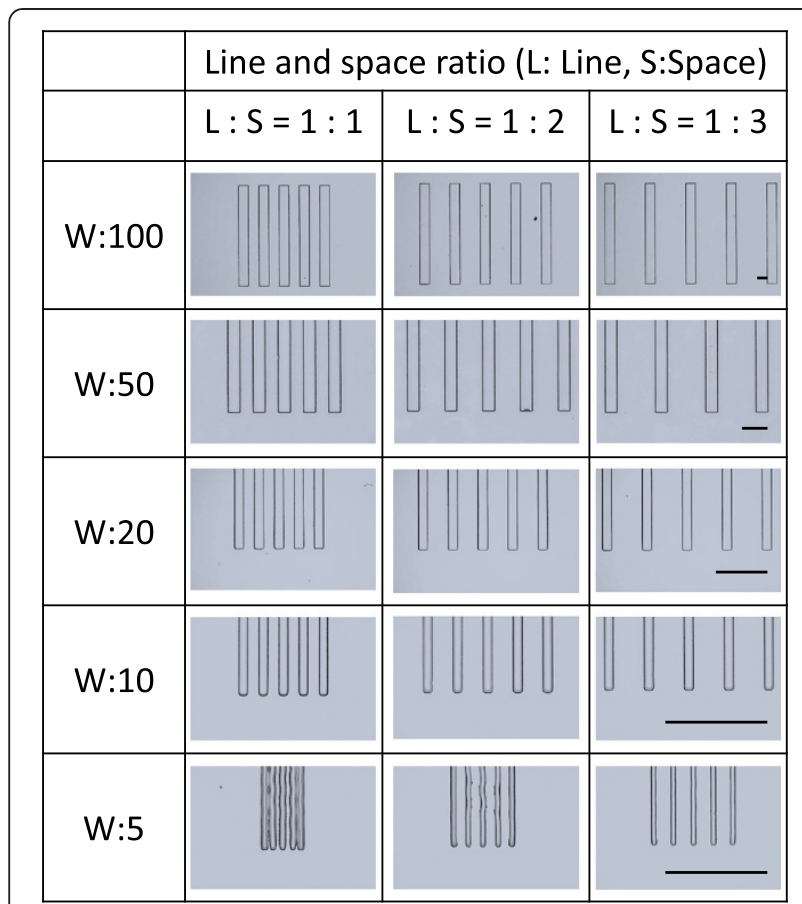

Figure 3 Images of the patterned Bioresist. W: Patterning width of Bioresist $[\mu \mathrm{m}]$. Scale bars are all $100 \mu \mathrm{m}$.

the valve because they have a solid and complex mechanical structure associated with the microfluidic channel. Pneumatic microvalves can be fabricated from soft materials such as polydimethylsiloxane (PDMS) and can reduce mechanical damage to cells [12-14]. However, each microvalve requires independent gas pressure control. This makes the whole system complex, and it is difficult to integrate many pneumatic microvalves into one chip. Magnetic microvalves can also be fabricated from soft materials. They are actuated by permanent magnets or electromagnets [15-18]. It is difficult to integrate many magnetic microvalves into one chip because the magnetic fields interfere with each other. Gel microvalves based on the thermoresponsive gels mixed with a solution in microfluidic channels are also reported such as poly(ethylene oxide $)_{\mathrm{x}^{-}}$ poly(propylene oxide $)_{y}$-poly (ethylene oxide $)_{x}$ and Poly- $(\mathrm{N}$ - isopropylacrylamide) (PNIPAAm) [19-26]. For example, by using the combination of the gel mixed solution and microelectrodes on the substrate of the microchannel, we can expect high-speed response of gel microvalves within several tens milliseconds $[19,24,25]$. These thermo-responsive gels are biocompatible material. The soft structure of such valves protects cells from mechanical damage [26]. Moreover, these valves can be actuated by local heating of the channel by microheaters or laser irradiation. The actuation mechanism is quite simple and can be easily integrated into a chip. However, conventional gel microvalves require mixing of thermoresponsive polymer into the culture medium.

In this paper, we propose a novel gel-valve using Bioresist. The gel-valve mainly consists of patterned gel and a microheater. Patterned gel is simply fabricated by photolithography of Bioresist, and the microheater is made using the lift off process. Such a simple fabrication process makes it possible to integrate many gel-valves into a chip. Furthermore, it has been already confirmed that Bioresist is biocompatible [26]. Gel-valves should therefore be suitable for cell manipulation on a chip.

\section{Gel-valve using Bioresist}

Bioresist has phase transition temperature because Bioresist consists of poly- $\mathrm{N}$-isopropyl acrylamide. Lower than phase transition temperature, Bioresist swells due to water absorption. Higher than phase transition temperature, Bioresist shrinks. Figure 1(a) shows the concept of the gel-valve using Bioresist. The gel-valves are patterned in the microchannel and actuated by local heating with a microheater fabricated below the patterned gel. Local heating is possible by reducing the width of the microheater at the heating point. The valve is normally closed, since Bioresist swells at the room temperature. When the valve is heated by applying electrical current to the microheater, it shrinks because of its thermal response, and the valve opens. The valve opens and closes because of the geometrical restriction of the microchannel, although Bioresist swells and shrinks isotropically. By using such actuation, we can perform several

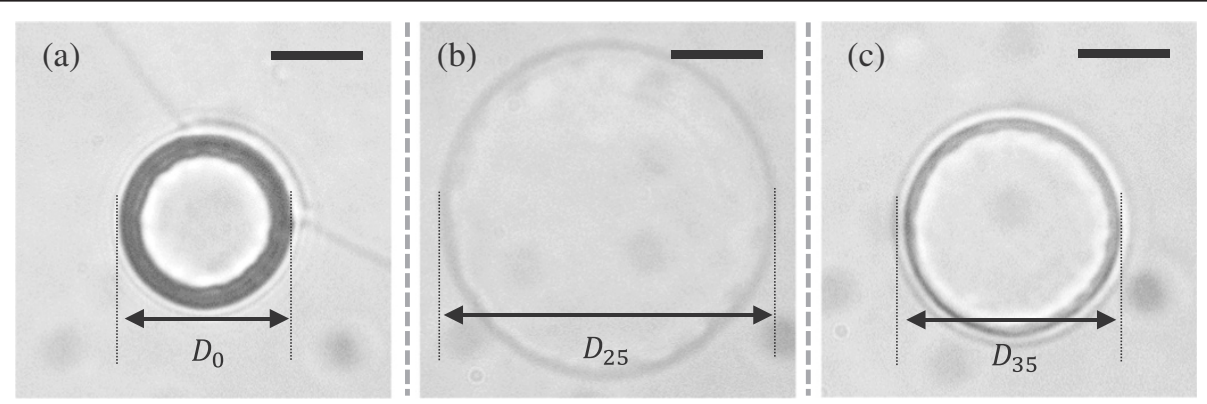

Figure 4 Examples of how Bioresist patterns shrink. (a) original pattern, (b) and (c) swollen pattern at 25 and 35 degrees, respectively. Scale bars are all $10 \mu \mathrm{m}$. 
microfluidic functions such as flow control, sorting, pumping, and cell loading as shown in Figure 1(b).

\section{Gel-valve fabrication process}

Figure 2 shows the fabrication process of a gel-valve, which mainly consists of patterned gel and a microheater. The valve is made in the microchannel, which is made by the PDMS cover. The microchannel and Bioresist are designed to have the same height; therefore, expansion of Bioresist is restricted by the top and bottom surfaces of the microfluidic channel. Eventually, we utilize the horizontal lateral motion for achieving valve function. The detailed fabrication process is as follows:

(a) Microheaters are patterned on the glass substrate using the lift-off process.

(b) Microchannels are fabricated using SU-8 (Nippon Kayaku Co. Ltd.).

(c) Bioresist is patterned by photolithography as a gel-valve.

(d) The molded PDMS cover is bonded with the glass layer, and the electrodes are attached.

\section{Results}

\section{Evaluation of Bioresist patterning resolution}

As Bioresist is photopatternable, we can create any arbitrary 2D shape using photolithography. We evaluated the patterning resolution of Bioresist by changing the line and space ratio of the pattern. In this evaluation, the thickness of Bioresist was fixed at $4 \mu \mathrm{m}$ for manipulation of Synechocystis sp. PCC 6803, which is a kind of cyanobacteria with a diameter of a few micrometers. Figure 3 shows examples of the fabricated patterns where the widths of the target patterns were 5, 10, 20, 50 and $100 \mu \mathrm{m}$, respectively. From Figure 3, we can see that the patterning resolution depends on the line and space ratio of the design, and that a larger line and space ratio gives higher resolution. For example, in the cases where the line and space ratios were $1: 1,1: 2$, and $1: 3$, the resolutions of the line patterns were 10, 10, and $5 \mu \mathrm{m}$, respectively. In the case where the line and space ratio was $1: 2$ and line width is $5 \mu \mathrm{m}$, unexposed resist remains on the pattern as a dissolved remainder. The reason the results depend on the design is one of the features of Bioresist. Bioresist patterns swell during development in the photofabrication process because the development process requires isopropyl alcohol (IPA) as an etchant, which causes expansion of Bioresist in the photo fabrication process.

\section{Evaluation of temperature dependent expansion of Bioresist}

We evaluated the expansion rate of Bioresist patterns by changing the temperature of the environment. The expansion rate is defined as shown in Equation 1

$$
\text { Expansion rate }=D_{t} / D_{0}
$$

where $D_{o}$ and $D_{t}$ are the diameter of patterned gel and the diameter of swollen gel at each temperature $t$ respectively. The environmental temperature was controlled by a thermo plate (TOKAI HIT. Co. Ltd., TP-CH110R) which is placed under the microfluidic chip. The photo of the shape of gel pattern was taken by the vision sensor attached to the microscope. We waited 10 minutes to confirm stabilization of each temperature for experiment. We measured the shape of patterned gel during temperature increasing phase, and then decreasing phase. The expansion rate is defined as the ratio between the swollen diameter at each temperature condition and the patterned diameter of the fabricated pillar. Figure 4 shows how Bioresist patterns change. The pattern diameter and the height are $20 \mu \mathrm{m}$ and $4 \mu \mathrm{m}$, respectively. From Figures 4(b) through (c), we can see that the shape of Bioresist is swollen at the low temperature condition, and that the shape shrinks with high temperature. Figure 5 shows the relationship between the expansion rate and

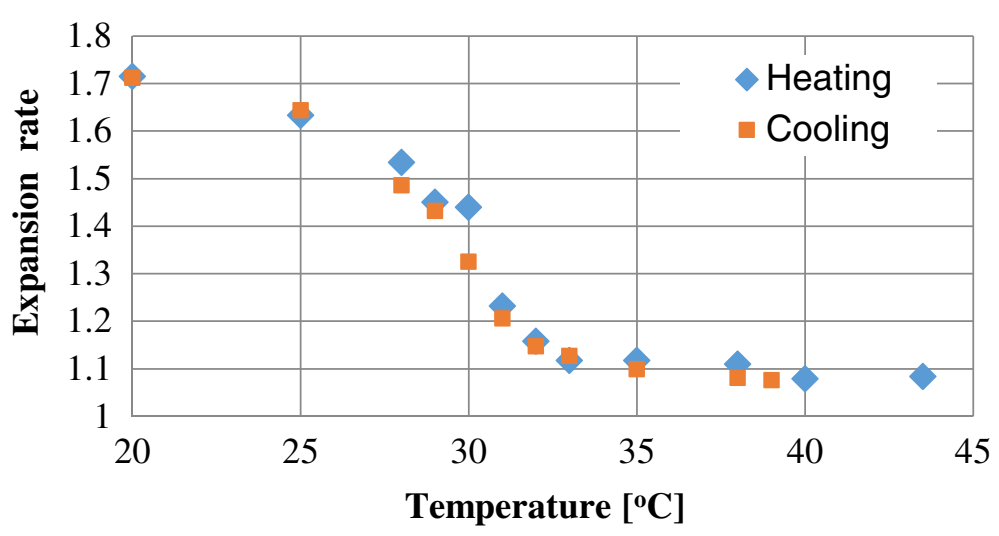

Figure 5 Relationship between expansion rate of Bioresist. 
temperature. From Figure 5, we can see that Bioresist has a phase transition, and the transition temperature is approximately $30^{\circ} \mathrm{C}$. Moreover, the hysteresis between the heating and cooling phases is small. The measured expansion rates were 1.1 through 1.7 with respect to temperature.

\section{Gel-valve evaluation}

We designed the gel-valve based on the results for patterning resolution and expansion rate. In this case, the width of the gel-valve structure and the gap between gels are designed as $20 \mu \mathrm{m}$ and $10 \mu \mathrm{m}$, respectively, as shown in Figure 6(a). Figures 6(b) and (c) show the fabricated valve and a scanning electron microscope (SEM) image of the gel-valve, respectively. The gel-valve is normally closed at room temperature (less than $30^{\circ} \mathrm{C}$ ), and opens when electrical current is applied to the microheater at the bottom of the patterned Bioresist. Figure 7 shows how the gel-valve moves by switching the heating on and off; the closing and opening movements are controlled by changing the electrical current to the microheater.

Figure 8 shows the relationship between the pressures in microchannel when the syringe pump was actuated. The pressure in the microchannel was measured using a pressure sensor (Copal electronics, PA-830) at the inlet of the channel. From 0 through 32 seconds the measured pressure increased, and then decreased with respect to time. This result corresponded to leakage that occurred due to the detachment of the PDMS cover from the glass substrate. From Figure 8, we confirmed that the leakage pressure of the microchannel using the gel-valves was $199.5 \mathrm{kPa}$.

Frequency characteristics of the gel-valve were evaluated as shown in Figure 9. Amplitude and frequency of the input current to the microheater were controlled by a function generator (NF Corporation, WF1974). The input signals were square waves; the currents were 33.2, 38.2 , and $43.7 \mathrm{~mA}$ respectively; and the frequency was changed from 0.1 to $10 \mathrm{~Hz}$. As a general tendency, the displacement of phase lag decreases with respect to the increase in frequency. When the applied current was $33.2 \mathrm{~mA}$, the gel-valve did not shrink faster than $1 \mathrm{~Hz}$ because the valve was not heated enough owing to the low current. On the other hand, when the applied current was $43.7 \mathrm{~mA}$, the valve was well-actuated up to $5 \mathrm{~Hz}$, but it did not swell enough at $10 \mathrm{~Hz}$ because the gel was not cooled enough at high frequency. From these results, we confirm that the amplitude of the applied current has a certain optimum value owing to the heating and cooling balance of the total system, and we set the current as $38.2 \mathrm{~mA}$ for the following experiments. In this case, we can actuate the gel-valve at $5 \mathrm{~Hz}$. In our experiment in 3.3 through 3.4, we

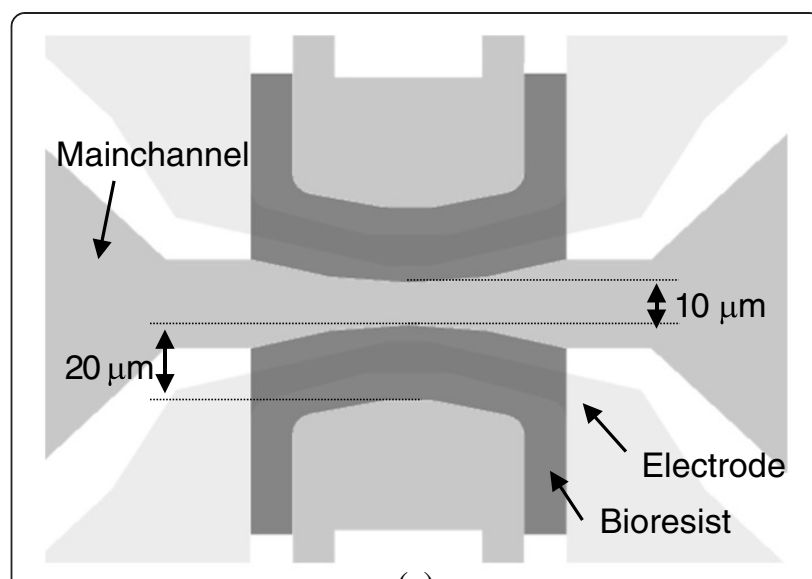

(a)

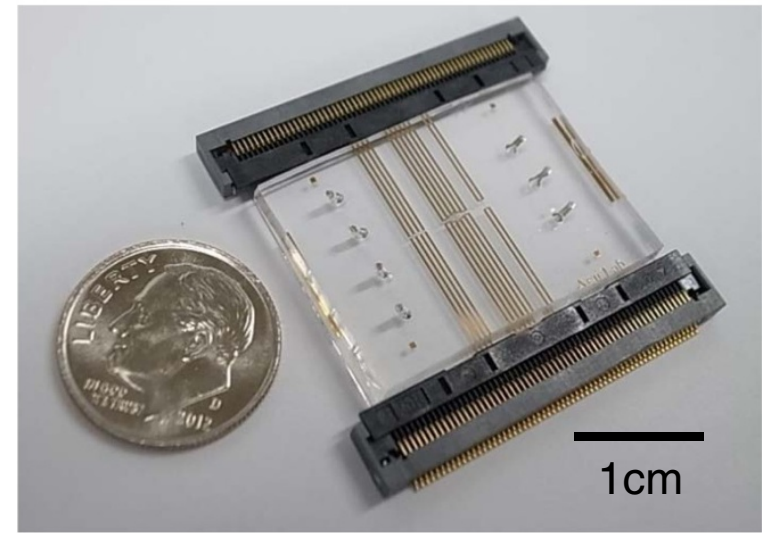

(b)

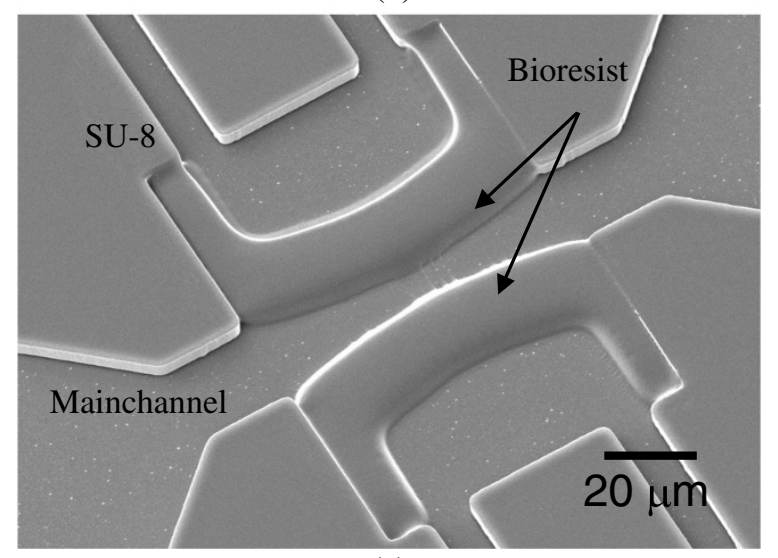

(c)

Figure 6 Images of design and fabricated devices. (a) A design image of gel- valve (b) A photo of fabricated chip. (c) SEM image of gel-valve.

fixed $\mathrm{pH}$ of the surrounding condition of the gelvalve using DI water. Since the expansion motion of the gel-actuator depends on its size and $\mathrm{pH}$ of the surrounding condition, we will investigate these effects on the response time with the desired culture condition of target cells in future $[27,28]$. 


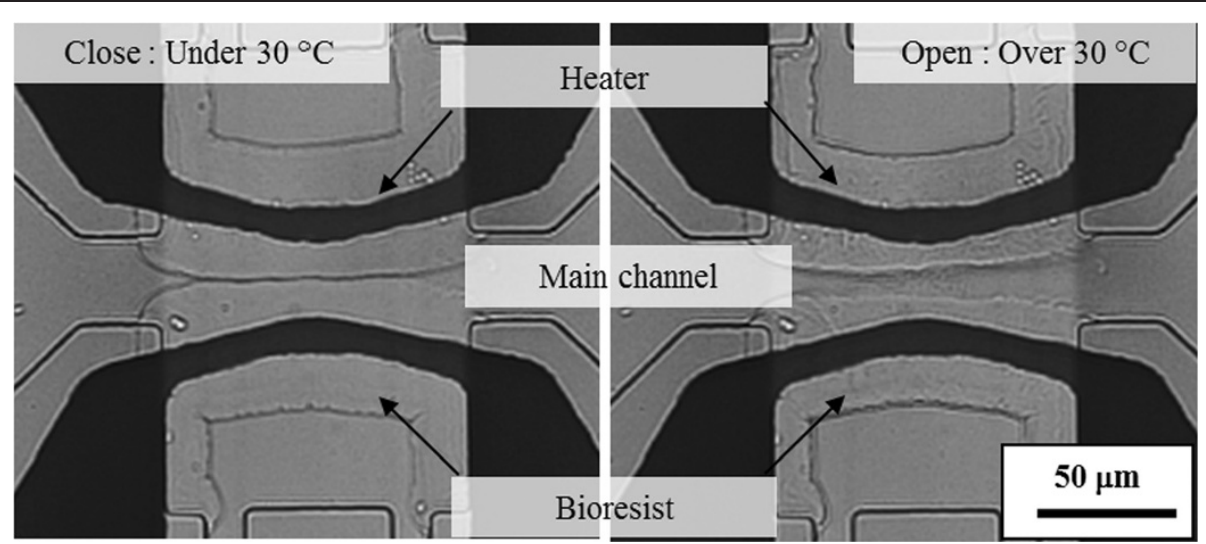

Figure 7 Demonstration of gel-valve.

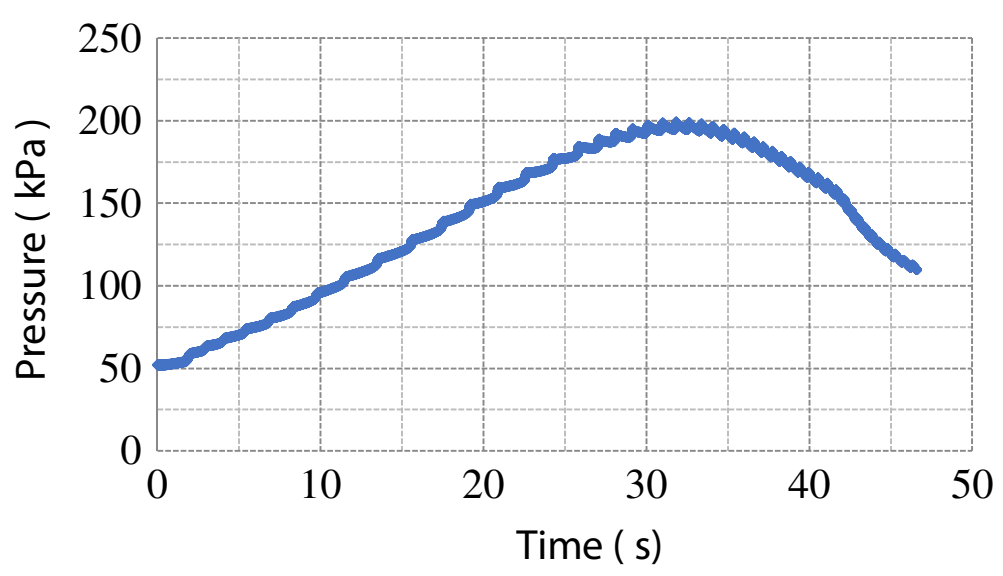

Figure 8 Measurement of leakage pressure.

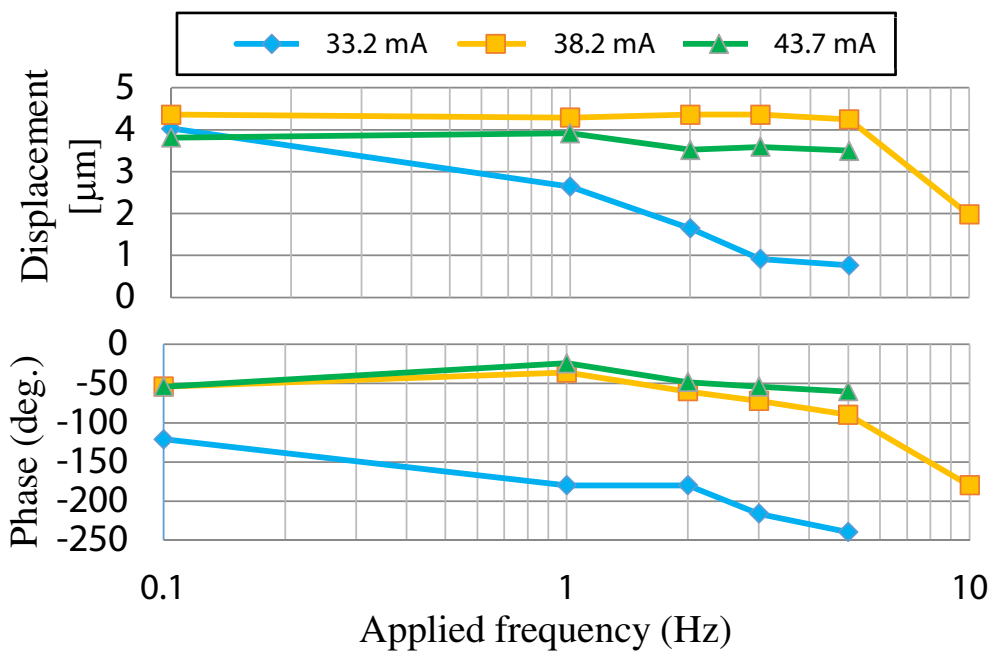

Figure 9 Frequency response of gel-valve. 


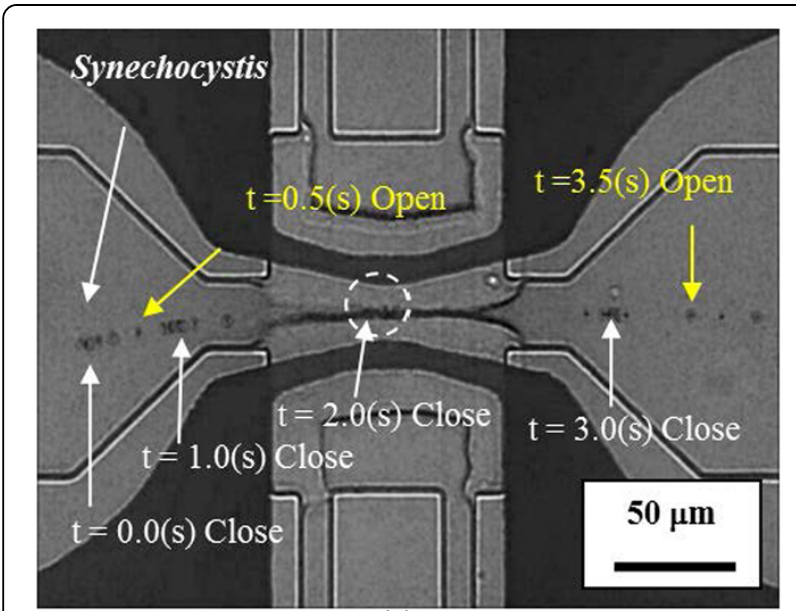

(a)

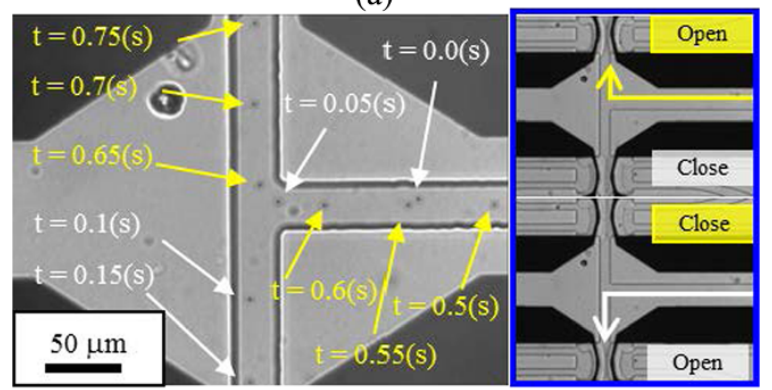

(b)

Figure 10 Merged figures of experiments. (a) A photo of valve experiments. (b) Photos of sorting experiments.

\section{Application: microvalve and cell sorter}

The gel-valve was evaluated as an on-chip flow control component. In this experiment, Synechocystis sp. PCC 6803, which is a kind of cyanobacteria with a diameter of a few micrometers, was used as a tracer. Figure 10(a) shows sequential photos of the movement of a Synechocystis bacterium flowing in the channel. The transportation of Synechocystis was controlled and stop-and-go motion was observed with respect to the closing and opening of the gel-valve; we could also observe Synechocystis through the valve (Additional file 1). Moreover, we demonstrated cell sorting by the combination of gel-valves shown in Figure 10(b). Two gel-valves were placed downstream of the branch of the microchannel. The flow was switched by changing the applied current to each valve, and the cells were sorted at the branch. From these results, we confirmed that the gel-valve works successfully, and that we can control the transportation of cells changing only the applied electrical current to the microheater.

\section{Discussion}

In this study, we focused on the evaluations of the gelvalve using Bioresist as an on-chip microfluidic component and demonstrated on-chip flow control using gel-valves. The proposed gel-valve is advantageous for integration as a microfluidic component in a chip, since the structure is quite simple. The gel-valves can be integrated using standard photolithography, which is suitable for mass production. Moreover, it can be driven with low power consumption. For example, we can actuate the gel-valve with $39.3 \mathrm{~mW}(38.2 \mathrm{~mA}$ and $1.03 \mathrm{~V}$ ) for open/close control. The gel-valve can be driven by a small battery, and we can easily set up a small system.

\section{Conclusions}

We proposed a gel-valve that can be highly integrated into a microfluidic chip. The gel-valve can be fabricated with $20 \mu \mathrm{m}$ resolution by standard photolithography and lift off processes. We can actuate the gel-valve at $5 \mathrm{~Hz}$ with $38.2 \mathrm{~mA}$. Furthermore, we demonstrated cell sorting by using two gel-valves fabricated near the branch of a microchannel on a chip. The target cell was successfully sorted. Since the proposed gel-valve can be actuated simply by local heating with a microheater with a power consumption of $39.3 \mathrm{~mW}$, we can easily apply the valve in microfluidic chips.

\section{Additional file}

Additional file 1: A movie of demonstration of gel-valve experiment with Synechocystis.

\section{Competing interests}

The authors declare that they have no competing interests.

\section{Authors' contributions}

All authors equally contributed. All authors read and approved the final manuscript.

\section{Acknowledgement}

This work is supported by a Grant-in-Aid for Scientific Research from the Ministry of Education, Culture, Sports, Science and Technology (23106002), the Japan Society for the Promotion of Science, and Nissan Chemical Industries, LTD.

\section{Author details}

${ }^{1}$ Nagoya University, Nagoya, Japan. ${ }^{2}$ Osaka University, Osaka, Japan. ${ }^{3} T o y a m a$ Industrial Technology Center, Toyama, Japan.

Received: 22 January 2014 Accepted: 24 May 2014 Published online: 30 August 2014

\section{References}

1. Wacogne B, Pieralli C, Roux C, Gharbi T (2008) Measuring the mechanical behaviour of human oocytes with a very simple SU-8 micro-tool. Biomed Microdevices 10:411-419. doi:10.1007/s10544-007-9150-7

2. Zare RN, Kim S (2010) Microfluidic platforms for single-cell analysis. Annu Rev Biomed Eng 12:187-201. doi:10.1146/annurev-bioeng-070909-105238

3. Lindström S, Andersson-Svahn H (2011) Miniaturization of biological assays overview on microwell devices for single-cell analyses. Biochim Biophys Acta 1810:308-316. doi:10.1016/j.bbagen.2010.04.009

4. Hagiwara M, Kawahara T, Yamanishi Y, Masuda T, Feng L, Arai F (2011) On-chip magnetically actuated robot with ultrasonic vibration for single cell manipulations. Lab Chip 11:2049-2054. doi:10.1039/c1lc20164f

5. Sakuma S, Arai F (2013) Cellular force measurement using a nanometricprobe-integrated microfluidic chip with a displacement reduction mechanism. J Robot Mechatronics 25:277-284 
6. Wheeler AR, Throndset WR, Whelan RJ , Leach AM, Zare RN, Liao YH, Farrell K, Manger ID, Daridon A (2003) Microfluidic device for single-cell analysis. Anal Chem 75:3581-3586

7. Li PC, Harrison DJ (1997) Transport, manipulation, and reaction of biological cells on-chip using electrokinetic effects. Anal Chem 69:1564-1568

8. Yamada M, Seki M (2005) Hydrodynamic filtration for on-chip particle concentration and classification utilizing microfluidics. Lab Chip 5:1233-1239. doi:10.1039/b509386d

9. Han J, Yeom J, Mensing G, Flachsbart B, Shannon MA (2012) Characteristics of electrostatic gas micro-pump with integrated polyimide passive valves. J Micromechanics Microengineering 22:095007. doi:10.1088/0960-1317/22/9/095007

10. Yildırm E, Arıkan MS, Külah H (2012) A normally closed electrostatic parylene microvalve for micro total analysis systems. Sensors Actuators A Phys 181:81-86. doi:10.1016/j.sna.2012.05.008

11. Anjewierden D, Liddiard GA, Gale BK (2012) An electrostatic microvalve for pneumatic control of microfluidic systems. J Micromechanics Microengineering 22:025019. doi:10.1088/0960-1317/22/2/025019

12. Hosokawa $K$, Maeda R (2000) A pneumatically-actuated three-way microvalve fabricated with polydimethylsiloxane using the membrane transfer technique. J Micromechanics Microengineering 10:415-420. doi:10.1088/0960-1317/10/3/317

13. Go JS, Shoji S (2004) A disposable, dead volume-free and leak-free in-plane PDMS microvalve. Sensors Actuators A Phys 114:438-444. doi:10.1016/j.sna.2003.12.028

14. Thuillier G, Malek CK (2005) Development of a low cost hybrid Si/PDMS multilayered pneumatic microvalve. Microsyst Technol 12:180-185. doi:10.1007/ s00542-005-0007-9

15. Bosch D, Heunhofer B, Muck G, Seldel H, Thumser U, Welser W (1993) A silicon microvalve with combined electromagnetic / electrostatic actuation. Sensors Actuators A Phys 38:684-692

16. Meckes A, Behrens J, Kayser O, Benecke W, Becker TH, Müller G (1999) Microfluidic system for the integration and cyclic operation of gas sensors. Sensors Actuators A Phys 76:478-483. doi:10.1016/S0924-4247(99)00060-6

17. Bae B, Kim N, Kee H, Kim SH, Lee Y, Lee S, Park K (2002) Feasibility test of an electromagnetically driven valve actuator for glaucoma treatment. J Microelectromechanical Syst 11:344-354. doi:10.1109/JMEMS.2002.800921

18. Fu C, Rummler Z, Schomburg W (2003) Magnetically driven micro ball valves fabricated by multilayer adhesive film bonding. J Micromechanics Microengineering 13:96-102

19. Stoeber B, Yang Z, Liepmann D, Muller SJ (2005) Flow control in microdevices using thermally responsive triblock copolymers. J Microelectromech Syst 14:207-213. doi:10.1109/JMEMS.2004.839330

20. Yamanishi Y, Teramoto J, Magariyama Y, Ishihama A, Fukuda T, Fumihito A (2009) On-chip cell immobilization and monitoring system using thermosensitive gel controlled by suspended polymeric microbridge. IEEE Trans Nanobioscience 8:312-317

21. Arai F, Ichikawa A, Fukuda T, Katsuragi T (2003) Isolation and extraction of target microbes using thermal sol-gel transformation. Analyst 128:547. doi:10.1039/b212919a

22. Ichikawa A, Arai F, Yoshikawa K, Uchida T, Fukuda T (2005) In situ formation of a gel microbead for indirect laser micromanipulation of microorganisms. Appl Phys Lett 87:191108. doi:10.1063/1.2126800

23. Arai F, Ng C, Maruyama H, Ichikawa A, El-Shimy H, Fukuda T (2005) On chip singlecell separation and immobilization using optical tweezers and thermosensitive hydrogel. Lab Chip 5:1399-1403. doi:10.1039/b502546j

24. Arakawa T, Shirasaki Y, Aoki T, Funatsu T, Shoji S (2007) Three-dimensional sheath flow sorting microsystem using thermosensitive hydrogel. Sensors Actuators A Phys 135:99-105. doi:10.1016/.jna.2006.06.074

25. Shirasaki Y, Tanaka J, Makazu H, Tashiro K, Shoji S, Tsukita S, Funatsu T (2006) On-chip cell sorting system using laser-induced heating of a thermoreversible gelation polymer to control flow. Anal Chem 78:695-701. doi:10.1021/ac0511041

26. Yokoyama Y, Umezaki M, Kishimura T, Tamiya E, Takamura Y (2011) Micro-and nano-fabrication of stimulus-responsive polymer using nanoimprint lithography. J Photopolymer Sci Technol 24:63-70

27. Tanaka T, Sato E, Hirokawa Y (1985) Critical kinetics of volume phase transition of gels. Phys Rev Lett 55:2455-2458

28. Zhang J, Peppas NA (2000) Synthesis and characterization of $\mathrm{pH}$ - and temperature-sensitive poly (methacrylic acid)/ poly ( $\mathrm{N}$-isopropylacrylamide) interpenetrating polymeric networks. Macromolecules 33:102-107

\section{Submit your manuscript to a SpringerOpen ${ }^{\circ}$ journal and benefit from:}

- Convenient online submission

- Rigorous peer review

- Immediate publication on acceptance

- Open access: articles freely available online

- High visibility within the field

- Retaining the copyright to your article

Submit your next manuscript at $\gg$ springeropen.com 